\title{
Formation of the cosmic-ray spectrum due to its propagation in the Galaxy
}

\author{
V. Kryvdyk ${ }^{\star}$ \\ Kyiv University, Department of Astronomy, Glushkova 6, 03022, Kyiv, Ukraine
}

Received 17 July 2002 / Accepted 13 December 2002

\begin{abstract}
A model of cosmic ray propagation is proposed to explain the knee of the cosmic ray energy spectrum in the energy range $E \sim 10^{14}-10^{17} \mathrm{eV}$. The numerous stellar winds (SW), ionized hydrogen regions (H-II) and supernova remnants (SNR) in the Galaxy are taken into account in this model. The gas density and the magnetic field in these regions are different from the interstellar gas density and the interstellar magnetic field. Therefore they act as scattering centres and magnetic traps for cosmic rays. It is shown that these regions influence cosmic ray propagation in the Galaxy. Our results show that the collision time between cosmic rays and the SNR, SW, and H-II regions is much less than the cosmic ray lifetime in standard models (Berezinskii et al. 1984; Ginzburg \& Syrovatskii 1963), in which only the nuclear interaction of the particles with interstellar gas is taken into account. Cosmic ray energies, and thus the cosmic ray spectrum, change due to interactions with these regions. Cosmic ray energy losses in these regions due to adiabatic cooling are comparable to the losses due to nuclear interaction with interstellar gas. It is therefore necessary to take these into account in galactic cosmic ray propagation models.
\end{abstract}

Key words. cosmic rays - diffusion - Galaxy: kinematics and dynamics

\section{Introduction}

Very simplified Galactic models were used in the first studies of cosmic-ray propagation in the Galaxy (Berezinskii et al. 1984; Ginzburg \& Syrovatskii 1963). Those studies took into account only the nuclear interactions of the particles with the homogeneous interstellar gas. Later, more realistic models were proposed (Erlykin \& Wolfendale 2001; Jonis et al. 2001; Houston et al. 1983; Li et al. 1983; Strong \& Moskalenko 1998; Tsao et al. 2001), which considered cosmic ray diffusion in the heterogeneous Galactic magnetic fields and in the inhomogeneous neutral and ionized hydrogen in the Galaxy.

Honda (1987) considered cosmic-ray propagation in Galactic magnetic fields (assumed to consist of the average field strength $\sim 3 \mu \mathrm{G}$, extending to $\sim 1 \mathrm{kpc}$ above and below the Galactic plane, with irregularities in the magnetic field strength of $\sim 1.5 \mu \mathrm{G}$ ). For the energy range $10^{16}-10^{18} \mathrm{eV}$, the gyroradius $(3-300 \mathrm{pc})$ is comparable with the scale of irregularities of the magnetic field. However, in this paper regions with strong magnetic fields such as stars, nebula, molecular clouds and Supernova Remnants were not considered.

Clay $(2000,2001)$ considered the propagation of cosmic rays with energies of about $10^{18} \mathrm{eV}$ from the vicinity of the Galactic centre in a Galactic magnetic field model with turbulent and regular components. He concluded that the turbulent field is crucial to resulting observations of cosmic rays. Clay (2002) also examined the relationship between the spectrum of

\footnotetext{
* e-mail: kryvdyk@mail.univ.kiev.ua
}

cosmic rays in their sources and the observed energy spectrum of cosmic rays. He concluded that the form of the spectrum in the sources differs greatly from the observed spectrum due to propagation effects.

Chandrang (2000) considered cosmic-ray diffusion and scattering on magnetized molecular clouds which act as magnetic mirrors for cosmic rays. In this paper it is shown that Galactic cosmic rays can be effectively confined through magnetic reflection by molecular clouds.

In our paper we consider a cosmic-ray propagation model which takes into account the presence in the Galaxy of stellar winds (SW), supernova remnants (SNR) and $\mathrm{H}-\mathrm{II}$ regions surrounding young O-B stars (H-II-O and H-II-B regions). These regions will influence the cosmic-ray propagation. The magnetic fields in these regions exceed the interstellar magnetic fields by an order magnitude and more (Allen et al. 2001; Bourke et al. 2001; Brogan et al. 2000; Crutcher et al. 1999; Dickel et al. 2000; Ginzburg \& Syrovatskii 1963; Gray et al. 1999; Gorbatskii 1977; Habing 1972; Ilovaisky \& Legueux 1972; Jun Byung \& Norman 1996; Lacey \& Duric 2001; Lozinskaia 1986; Schleuning et al. 2000; Shklovskii 1976; Woltjer 1972). Also, these regions are dynamically unstable and gas expands from their centers. The cosmic rays scatter in these regions on the magnetic heterogeneity and their energy decreases as a result of adiabatic cooling. We will consider these topics in this paper.

We assume that the Galaxy consists of interstellar gas, regions such as SW, SNR, H-II-O, H-II-B and relativistic cosmic 
Table 1. The value $T_{\mathrm{c} i}$ (years) for various regions of Galaxy.

\begin{tabular}{llll}
\hline \hline Regions & $N_{i}$ & $R_{i}, \mathrm{~cm}$ & $T_{\mathrm{c} i}$, \\
\hline H-II-O & $10^{4}$ & $1 \times 10^{20}$ & 14000 \\
H-II-B & $10^{5}$ & $3 \times 10^{19}$ & 15520 \\
Old SNRs & $10^{4}$ & $4 \times 10^{19}$ & 87300 \\
SW & $10^{11}$ & $1.5 \times 10^{15}$ & $6.2 \times 10^{6}$ \\
\hline
\end{tabular}

rays. We also assume that the Galaxy has the form of the rotation ellipsoid with semi-axis $a=d=15 \mathrm{kps} \approx 4.5 \times 10^{22} \mathrm{~cm}$, $b=150 \mathrm{ps} \approx 4.5 \times 10^{20} \mathrm{~cm}$ with a homogeneous star distribution.

\section{Cosmic-ray propagation in the Galaxy}

\subsection{Cosmic ray scattering on the SNR, SW and $\mathrm{H}$-II regions}

In our model, the cosmic rays are accelerated in supernova remnants. They remain for some time $T_{s}$ in the SNRs and later emerge into interstellar space. Propagating in the Galaxy, the cosmic rays collide with SNR, SW and $\mathrm{H}-\mathrm{II}$ regions, which can be considered as heterogeneous magnetic fields against a background of weaker regular interstellar magnetic fields. The cosmic rays scatter on these regions, and some of them can be captured there.

The mean time for collisions between the cosmic rays and the heterogeneous magnetic fields of type $i$ is $T_{\mathrm{c} i}=1 / \Omega_{i} \rho_{i} v$. (Ginzburg \& Syrovatskii 1963). Here $v$ is the particle velocity, $\Omega_{i}=\pi R_{i}^{2}$ is the mean cross-section of the magnetic heterogeneities, $R_{i}$ are their radii and $\rho_{i}$ is their density in the Galaxy. For a homogeneous distribution of magnetic heterogeneities in the Galaxy, their density $\rho_{i}=N_{i} / V_{\mathrm{G}}$, where $N_{i}$ is the number of heterogeneities in the Galaxy.

For relativistic particles $v \approx c$ and

$T_{\mathrm{c} i}=1.4 \times 10^{48} \frac{1}{R_{i}^{2} N_{i}}$ years.

The values $T_{\mathrm{c} i}$ for the various regions in Galaxy (for regions H-II-O and H-II-B surrounding O and B stars, SNR and SW solar type - with $R_{i} \approx 100$ a.u. $=1.5 \times 10^{15} \mathrm{~cm}$ ) are given in Table 1. The values $N_{i}$ and $R_{i}$ were taken from papers (Hundhausen 1976; Shklovskii 1976; Sobolev 1985; Woltjer 1972).

It follows from Table 1 that the collision times of the cosmic rays with SNRs, SW and H-II regions are much smaller than the cosmic-ray lifetime due to nuclear interactions with interstellar gas (this time $T_{n} \approx 10^{8}$ years in the model Ginzburg \& Syrovatskii 1963). The physical conditions in these regions differ considerably from the conditions in the interstellar medium. Firstly, these regions are dynamically unstable with expanding gas. Whilst in these regions, the cosmic rays lose energy due to adiabatic cooling. Secondly, the magnetic field in these regions is heterogeneous. Propagating in the Galaxy, cosmic rays will scatter on these regions. For some cosmic rays, these regions can be magnetic traps.

We consider cosmic-ray propagation in the Galaxy taking into account SNR, SW and $\mathrm{H}-\mathrm{II}$ regions. These regions
Table 2. The values $L_{i}(\mathrm{~cm})$ for SW, SNR and H-II regions.

\begin{tabular}{llllll}
\hline \hline$E(\mathrm{eV})$ & $r_{H_{i}}(\mathrm{~cm})$ & H-II-O & H-II-B & SNR & SW \\
\hline $10^{8}$ & $3 \times 10^{9}$ & $1 \times 10^{22}$ & $1 \times 10^{22}$ & $2 \times 10^{23}$ & $2 \times 10^{25}$ \\
$10^{18}$ & $3 \times 10^{18}$ & $1 \times 10^{22}$ & $1 \times 10^{22}$ & $2 \times 10^{23}$ & $7 \times 10^{33}$ \\
$10^{19}$ & $3 \times 10^{20}$ & $2 \times 10^{22}$ & $3 \times 10^{23}$ & $1 \times 10^{25}$ & $7 \times 10^{35}$ \\
$10^{20}$ & $3 \times 10^{21}$ & $2 \times 10^{24}$ & $3 \times 10^{25}$ & $1 \times 10^{27}$ & $7 \times 10^{37}$ \\
$10^{21}$ & $3 \times 10^{22}$ & $2 \times 10^{26}$ & $3 \times 10^{27}$ & $1 \times 10^{29}$ & $7 \times 10^{39}$ \\
\hline
\end{tabular}

can be considered as the magnetic heterogeneities with field strengths $H_{i}$ and radii $R_{i}$, scattering the cosmic rays. Between these regions are the weaker interstellar regular magnetic fields with $H_{\mathrm{G}} \approx 1 \mu \mathrm{G}$. In such a system, the diffusion coefficient is (Dorman 1975; Ginzburg \& Syrovatskii 1963)

$D_{i}=v L_{i} / 3$,

where $L_{i}$ is the mean free path.

For cosmic rays in the Galactic disk, the mean free path (Dorman 1975; Ginzburg \& Syrovatskii 1963)

$L_{i} \approx\left(4 \times 10^{66} / N_{i}\right) R_{i}^{-4}\left(r_{H_{i}}^{2}+R_{i}^{2}\right)$.

Here $r_{H_{i}}(\mathrm{~cm})=E(\mathrm{eV}) / 300 H_{i}(\mathrm{G})$ is the Larmor radius.

Substituting (3) into (2), we obtain the diffusion coefficient for the relativistic particles $(v \approx c)$

$D_{i} \approx\left(4 \times 10^{56} / N_{i}\right) R_{i}^{-4}\left(r_{H_{i}}^{2}+R_{i}^{2}\right)$.

The cosmic rays diffuse out from the Galaxy and the cosmicray flux at a distance $R$ from the Galactic centre is (Dorman 1975; Ginzburg \& Syrovatskii 1963)

$S_{\mathrm{d} i} \propto 4 \pi R D_{i} n$.

The outflow time of the cosmic rays from Galaxy due to the diffusion is

$T_{\mathrm{d} i}=n V_{\mathrm{G}} / S_{\mathrm{d} i}$

Here $n V_{\mathrm{G}}$ is the total number of cosmic rays particles in the Galaxy.

The total cosmic-ray energy $W$ in the Galaxy decreases to

$U_{\mathrm{d} i}=W / T_{s i}$.

The values $L_{i}$ for SW, SNR and $\mathrm{H}-\mathrm{II}$ regions are given in Table 2 where the magnetic fields were chosen as $H_{i}=10 \mu \mathrm{G}$ for all regions.

From these results we see that the mean free path is less than the dimensions of Galaxy only for charged particles with energy $E \leq 10^{19} \mathrm{eV}$ in H-II-O regions. Therefore these regions will scatter such particles. The mean free path in H-II regions for particles with energies $E>10^{19} \mathrm{eV}$ is larger than Galactic dimensions. Therefore the scattering of these particles can be neglected.

For stellar winds, the value $L_{i} \geq 2 \times 10^{25} \mathrm{~cm}$ for all particles with energy $E \geq 10^{8} \mathrm{eV}$. For young SNRs $R_{s \mathrm{o}} \approx 10^{15} \mathrm{~cm}$, $H_{s o} \approx 1 \mathrm{G}$, and the value $L_{i} \geq 10^{34} \mathrm{~cm}$ for all particles with energy $E \geq 10^{8} \mathrm{eV}$. These values exceed the Galactic radius. 
Therefore cosmic ray scattering on SW and young SNR can be neglected for such particles.

Thus, H-II regions are the effective scattering centres for the Galactic cosmic rays with the energy $E \leq 10^{19} \mathrm{eV}$. The diffusion coefficient is $D_{i} \approx 10^{32} \mathrm{~cm}^{2} \mathrm{~s}^{-1}$, and the cosmic-ray flux at a distance $R$ from the Galactic centre is $S_{\mathrm{d}} \approx 4 \pi R D_{i} n \approx$ $1.2 \times 10^{23} R$. The cosmic-ray flux in the polar regions of the Galaxy $\left(R_{\mathrm{p}} \approx 5 \times 10^{22} \mathrm{~cm}\right)$ is $S_{\mathrm{dp}} \approx 6 \times 10^{45} \mathrm{part} \mathrm{s}^{-1}$. These value exceed by ten time the value $S_{\mathrm{d}}$ in the halo model (Ginzburg $\&$ Syrovatskii 1963) $\left(S_{\mathrm{dg}} \approx\left(10^{43}-10^{45}\right)\right.$ part s $\left.{ }^{-1}\right)$, in which the scattering centers were considered to be the heterogeneities of the galactic magnetic fields with radii $R_{\mathrm{g}} \propto\left(3 \times 10^{19}-3 \times\right.$ $\left.10^{20}\right) \mathrm{cm}$.

The outflow time from the Galactic disk is $T_{\mathrm{dp}}=n V_{\mathrm{Gp}} / S_{\mathrm{dp}}$ ( $V_{\mathrm{Gp}} \approx 4 \times 10^{66} \mathrm{~cm}^{3}$ is the volume of the Galactic disk). The value $T_{\mathrm{dp}} \approx 10^{5}$ years, i.e. the outflow time from the Galactic disk is a thousand times smaller than the outflow time in the standard halo model for which $T_{\mathrm{g}} \approx 3 \times 10^{8}$ years (Ginzburg $\&$ Syrovatskii 1963). This requires a hundred times greater cosmic-ray power in the Galaxy $U_{\mathrm{d} i}=W_{\mathrm{Gp}} / T_{\mathrm{dp}}=\omega V_{\mathrm{Gp}} / T_{\mathrm{dp}} \approx$ $10^{42} \mathrm{erg} / \mathrm{s}$ in comparison with Ginzburg-Syrovatskiy model for which $U_{\mathrm{g} i} \approx 3 \times 10^{40} \mathrm{erg} / \mathrm{s}$. Here $\omega \approx 10^{-12} \mathrm{erg} / \mathrm{cm}^{3}$ is the energy density of the cosmic rays in the Galaxy.

\subsection{Energy losses of cosmic rays in the Galaxy}

It is assumed that supernova remnants are the main cosmicray sources in the Galaxy (Berezinskii et al. 1984; Erlykin \& Wolfendale 2000; Ginzburg \& Syrovatskii 1963; Jonis et al. 2001; Strong \& Moskalenko 1998; Tsao et al. 2001). The cosmic rays are accelerated in pulsar magnetospheres and supernova shocks. The accelerated particles spend some time $T_{\mathrm{o}}$ in SNRs and later on they go out into interstellar space. After the some time $T$ the cosmic rays escape the Galaxy and emerge into intergalactic space.

SNRs are magnetic traps for the cosmic rays. The charged particles lose energy in these traps as a result of bremsstrahlung losses in the magnetic fields, adiabatic cooling and nuclear interactions with the gas. The dimensions of the traps, and their magnetic fields, evolve. Therefore, the particle lifetime $T_{s}$ in SNRs depends on the age of the SNRs $t$, i.e. $T_{s}=T_{s}(E, t)$. The cosmic-ray spectrum changes due to propagation in the SNRs. The particles emerge into interstellar space with a changed spectrum in comparison with the spectrum in the pulsar magnetosphere or the shock wave. Later, this spectrum will further change due to propagation in the interstellar medium. After time $T$, the particles escape the Galaxy, which itself can be considered as a magnetic trap with stable parameters, i.e. $T=T(E)$.

So, the observed cosmic ray spectrum is formed due to propagation both in the interstellar matter and in SNRs. The cosmic rays lose energy in consequence of 1) bremsstrahlung losses in magnetic fields, 2) adiabatic cooling in expanding regions, 3) nuclear interactions with the gas and 4) cosmic-ray outflow from the regions. We will consider these processes in more detail and estimate the energy losses for various mechanisms in SNRs and interstellar matter.
The energy losses for charged particle in a region with volume $V_{i}$ and particle concentration $n$ due to a mechanism $k$ is given by

$$
\left(\frac{\mathrm{d} E}{\mathrm{~d} t}\right)_{k}=n V_{i}\left(\frac{\mathrm{d} E}{\mathrm{~d} t}\right)_{\mathrm{o} k}
$$

Here $\left(\frac{\mathrm{d} E}{\mathrm{~d} t}\right)_{\mathrm{o} k}$ is the energy loss for a particle due to the mechanism $k$.

The values $\left(\frac{\mathrm{d} E}{\mathrm{~d} t}\right)_{\mathrm{o} k}$ for nuclear interactions, bremsstrahlung losses, adiabatic losses and the cosmic-ray outflow from the region are (Ginzburg \& Syrovatskii 1963)

$$
\begin{aligned}
& \left(\frac{\mathrm{d} E}{\mathrm{~d} t}\right)_{\mathrm{oN}}=c \sigma \rho_{\mathrm{g} i} E, \\
& \left(\frac{\mathrm{d} E}{\mathrm{~d} t}\right)_{\mathrm{OM}}=c_{1} H_{i}^{2} E^{2} \\
& \left(\frac{\mathrm{d} E}{\mathrm{~d} t}\right)_{\mathrm{OA}}=v_{i} E / R_{i} \\
& \left(\frac{\mathrm{d} E}{\mathrm{~d} t}\right)_{\mathrm{OE}}=E / T_{i}
\end{aligned}
$$

Here $v_{i}, R_{i}, H_{i}, \rho_{\mathrm{g} i}$ are the expansion speed, radius, magnetic field and the gas density in the region $i ; T_{i}$ is the lifetime of the particles in region $i$; $\sigma$ is the cross-section for nuclear interaction of the cosmic rays with the gas; constant $c_{1} \approx 3 \times 10^{-28}$; $c=3 \times 10^{10} \mathrm{~cm} / \mathrm{s}$ is the velocity of light.

The relationships between the energy losses for the different mechanisms in the interstellar matter and in the different Galactic regions can be written as

$$
\begin{aligned}
& J_{1}=\left(\frac{\mathrm{d} E}{\mathrm{~d} t}\right)_{\mathrm{OE}} /\left(\frac{\mathrm{d} E}{\mathrm{~d} t}\right)_{\mathrm{oN}} \\
& J_{2}=\left(\frac{\mathrm{d} E}{\mathrm{~d} t}\right)_{\mathrm{oE}} /\left(\frac{\mathrm{d} E}{\mathrm{~d} t}\right)_{\mathrm{oM}}, \\
& J_{3}=\left(\frac{\mathrm{d} E}{\mathrm{~d} t}\right)_{\mathrm{oE}} /\left(\frac{\mathrm{d} E}{\mathrm{~d} t}\right)_{\mathrm{oA}}
\end{aligned}
$$

The total energy losses of the cosmic rays due to the adiabatic cooling in all the expanding Galactic regions (SNRs, SW, H-II regions) are

$$
\left(\frac{\mathrm{d} E}{\mathrm{~d} t}\right)_{\mathrm{oAG}}=v_{i} N_{i} E V_{\mathrm{G}} / R_{i}
$$

Here $N_{i}$ is the number of regions $i$ in the Galaxy, $V_{\mathrm{G}}$ is the Galactic volume.

From Eqs. (8)-(11) we obtain for the interstellar matter

$$
\begin{aligned}
& J_{1 \mathrm{G}}=\left(c \rho_{\mathrm{gG}} \sigma T_{\mathrm{G}}\right)^{-1}, \\
& J_{2 \mathrm{G}}=\left(c_{1} H_{\mathrm{G}}^{2} E T_{\mathrm{G}}\right)^{-1}, \\
& J_{3 \mathrm{G}}=V_{\mathrm{G}} R_{i}\left(N_{i} V_{i} v_{i} T_{\mathrm{G}}\right)^{-1} .
\end{aligned}
$$

Here $T_{\mathrm{G}}$ is the cosmic-ray lifetime in the Galaxy. For the Galaxy $V_{\mathrm{G}} \approx 4 \times 10^{66} \mathrm{~cm}^{3}, \rho_{\mathrm{gG}} \approx 1 \mathrm{~cm}^{-3}, H_{\mathrm{G}} \approx 1 \mu \mathrm{G}$, $T_{\mathrm{G}} \approx 3 \times 10^{13} \mathrm{~s}, \sigma \approx 2 \times 10^{-26} \mathrm{~cm}^{2}$ (Ginzburg \& Syrovatskii 1963; Sobolev 1985). 
For these parameters of the interstellar matter

$J_{1 \mathrm{G}} \approx 6$

$J_{2 \mathrm{G}} \approx 10^{25} / E$,

$J_{3 \mathrm{G}} \geq 7$.

It follows from these results that all values $J_{1 \mathrm{G}}>1, J_{2 \mathrm{G}}>1$, $J_{3 \mathrm{G}}>1$ for particles with $E \leq 10^{21} \mathrm{eV}$, therefore we can assume that the cosmic rays lose their energy mainly due to their outflow from the Galaxy.

For SNRs

$J_{1 s}=\left(c T_{s} \rho_{\mathrm{g} s} \sigma_{s}\right)^{-1}$,

$J_{2 s}=\left(c_{1} H_{s}^{2} E T_{s}\right)^{-1}$,

$J_{3 s}=R_{s}\left(v_{s} T_{s}\right)^{-1}$.

For SNRs $10^{15} \mathrm{~cm} \leq R_{s} \leq 10^{20} \mathrm{~cm}, \quad 10 \mu \mathrm{G} \leq H_{s} \leq 1 \mathrm{G}$, $5 \times 10^{6} \mathrm{~cm} / \mathrm{s} \leq v_{s} \leq 5 \times 10^{8} \mathrm{~cm} / \mathrm{s}, \rho_{\mathrm{SNR}} \approx 10 \mathrm{~cm}^{-3}$ (Ginzburg \& Syrovatskii 1963; Gorbatskii 1977; Habing 1972; Gray et al. 1999; Ilovaisky \& Legueux 1972; Jun Byung \& Norman 1996; Lacey \& Duric 2001; Pacini \& Salvati 1973; Schleuning et al. 2000; Shklovskii 1976; Woltjer 1972) For such SNRs

$J_{1 s}=2 \times 10^{14} / T_{s}$,

$3 \times 10^{27} / E T_{s} \leq J_{2 s} \leq 3 \times 10^{37} / E T_{s}$

$2 \times 10^{6} / T_{s} \leq J_{3 s} \leq 2 \times 10^{13} / T_{s}$.

If we assume that the particle lifetime $T_{s}$ in the SNR equals the lifetime $T_{s}=R_{s} / v_{s}$, we obtain

$4 \times 10^{2} \leq J_{1 s} \leq 10^{9}$,

$2 \times 10^{21} / E \leq J_{2 s} \leq 6 \times 10^{24} / E$,

$J_{3 s}>1$.

In reality $T_{i}<R_{i} / v_{i}$, therefore inequality (16) will apply during all the SNR lifetime. From (16), it follows that $J_{i}>1$ for all stages of SNR evolution. It follows that the cosmic-ray energy in SNRs decreases mainly due to their outflow from SNRs.

\subsection{The adiabatic cooling of cosmic rays in SW, SNRs and $\mathrm{H}-\mathrm{Il}$ regions}

The charged particles lose energy due to adiabatic cooling whilst propagating in the expanding SW, SNRs and H-II regions.

The adiabatic losses for all regions of type $i$ in the Galaxy (Kryvdyk 1978)

$\left(\frac{\mathrm{d} E}{\mathrm{~d} t}\right)_{i}=\frac{v_{i}}{R_{i}} N_{i} V_{i} n(E) E$.

For spherically symmetric regions $V_{i}=\frac{4}{3} \pi R_{i}^{3}$ and for a homogeneous cosmic-ray distribution in Galaxy, we can write

$\left(\frac{\mathrm{d} E}{\mathrm{~d} t}\right)_{i}=\frac{4}{3} \pi \omega v_{i} N_{i} R_{i}^{2}$.

The sum of losses of cosmic-ray energy in all expanding Galactic regions is

$\left(\frac{\mathrm{d} E}{\mathrm{~d} t}\right)_{\mathrm{G}}=\sum_{i}\left(\frac{\mathrm{d} E}{\mathrm{~d} t}\right)_{i}$.
Table 3. The values $(\mathrm{d} E / \mathrm{d} t)_{i}$ for $\mathrm{SW}$, old SNRs and H-II regions in the Galaxy.

\begin{tabular}{lllll}
\hline \hline Regions & $N_{i}$ & $R_{i}, \mathrm{~cm}$ & $v_{i}, \mathrm{~cm} / \mathrm{s}$ & $\left(\frac{\mathrm{d} E}{\mathrm{~d} t}\right)_{i}, \mathrm{erg} / \mathrm{s}$ \\
\hline H-II-O & $10^{4}$ & $1 \times 10^{20}$ & $2 \times 10^{6}$ & $1 \times 10^{39}$ \\
H-II-B & $10^{5}$ & $3 \times 10^{19}$ & $2 \times 10^{6}$ & $6 \times 10^{38}$ \\
SNRs & $10^{4}$ & $4 \times 10^{19}$ & $2 \times 10^{7}$ & $1 \times 10^{39}$ \\
SW & $10^{11}$ & $2 \times 10^{15}$ & $4 \times 10^{7}$ & $1 \times 10^{37}$ \\
\hline
\end{tabular}

Numerical values of $(\mathrm{d} E / \mathrm{d} t)_{i}$ for SW, old SNRs and H-II regions in the Galaxy are given in Table 3.

The values $N_{i}, R_{i}, v_{i}$ are given in papers (Hundhausen 1976; Ilovaisky \& Legueux 1972; Lozinskaia 1986; Pacini \& Salvati 1973; Shklovskii 1976; Sobolev 1985; Woltjer 1972).

The total cosmic-ray energy losses in SW, SNRs and H-II regions in Galaxy are

$\left(\frac{\mathrm{d} E}{\mathrm{~d} t}\right)_{\mathrm{G}} \approx 3 \times 10^{39} \mathrm{erg} / \mathrm{s}$.

These losses are not less than the losses due to nuclear collisions of cosmic rays with the interstellar gas $(\mathrm{d} E / \mathrm{d} t)_{N} \approx(3 \times$ $\left.10^{38}-3 \times 10^{39}\right) \mathrm{erg} / \mathrm{s}$ (Ginzburg \& Syrovatskii 1963). Therefore these losses must be taken into account in models of cosmic ray propagation in the Galaxy. The total source power of cosmic rays in the Galaxy must then be increased twofold compared with the standard model.

\section{Cosmic-ray spectrum transformation due to propagation}

From the above results it follows that the cosmic-ray spectrum changes in the main due to outflow from SNRs and the Galaxy. Taking into account this result, we now consider the formation of the cosmic ray spectrum due to propagation in the Galaxy.

The cosmic-ray concentration in the interstellar medium can be written

$n_{\mathrm{G}}(E, t)=Q_{\mathrm{G}}(E, t) T_{\mathrm{G}}(E)$.

Here $Q_{\mathrm{G}}(E, t)$ is the source power of the cosmic rays in the Galaxy.

A similar value can be written for each cosmic-ray source of type $i$

$n_{i}(E, t)=Q_{i}(E, t) T_{i}(E, t)$

where $Q_{i}(E, t)$ is the power of source type $i$. This is the number of particles which this source injects into interstellar space per unit time. $T_{i}(E, t)$ is the cosmic-ray lifetime in this source.

Suppose that in the Galaxy there are $N_{i}$ sources of type $i$ with volume $V_{i}$ and power $Q_{i}(E, t)$. In order to have constant cosmic-ray concentration in the Galaxy, it is necessary to satisfy the condition

$Q_{\mathrm{G}} V_{\mathrm{G}}=\sum_{i=1}^{N_{i}} Q_{i} V_{i}$ 
From Eqs. (21)-(23) we obtain for the cosmic-ray concentration in the Galaxy

$n_{\mathrm{G}}=\sum_{i=1}^{N_{i}} n_{i} \frac{V_{i}}{V_{\mathrm{G}}} \frac{T_{\mathrm{G}}}{T_{i}}$.

For our model in which the main cosmic-ray sources in the Galaxy are SNRs,

$n_{\mathrm{G}}=N_{s} n_{s} \frac{V_{s}}{V_{\mathrm{G}}} \frac{T_{\mathrm{G}}}{T_{s}}$,

where $N_{s}$ is the number SNRs in the Galaxy.

We now consider in more detail the dependences $T_{\mathrm{G}}=$ $T_{\mathrm{G}}(E)$ and $T_{s}=T_{s}(E, t)$.

For a magnetic trap with radius $R_{i}$, having a magnetic field $H_{i}$ and magnetic heterogeneities with radius $l_{i}$, the particle lifetime can be written (Dorman 1975)

$T_{i}(E) \propto E^{-\mu_{i}(E)}$,

where

$\begin{array}{ll}\mu_{i}(E)=0 & \text { for } \quad R_{i}>>l_{i} \geq r_{H_{i}}, \\ \mu_{i}(E)=\mu_{i} & \text { for } \quad R_{i}>>r_{H_{i}} \geq l_{i}, \\ \mu_{i}(E)=0 & \text { for } \quad r_{H_{i}}>>R_{i} \geq l_{i} .\end{array}$

Here $r_{H_{i}}(\mathrm{~cm})=E(\mathrm{eV}) / 300 H_{i}(\mathrm{G})$ is Larmor radius.

For the Galaxy as the magnetic trap $H_{\mathrm{G}} \approx 1 \mu \mathrm{G}, R_{\mathrm{G}} \approx$ $10^{22} \mathrm{~cm}, l_{\mathrm{G}} \approx 3 \times 10^{19} \mathrm{~cm}$.

For these parameters

$$
\begin{array}{ll}
\mu_{\mathrm{G}}(E)=0 & \text { for } E \leq 3 \times 10^{15} \mathrm{eV}, \\
\mu_{\mathrm{G}}(E)=\mu_{\mathrm{G}} & \text { for } 3 \times 10^{15} \mathrm{eV}<E \leq 3 \times 10^{17} \mathrm{eV}, \\
\mu_{\mathrm{G}}(E)=0 & \text { for } E>3 \times 10^{17} \mathrm{eV} .
\end{array}
$$

Now we consider cosmic ray propagation in supernova remnants. SNRs are magnetic traps with unstable parameters. However, at any fixed moment in time they can be considered as stable traps. Therefore the function (26) can be used for the estimation of the particle lifetime in SNRs at a given time in their evolution. The SNR radius increases with time, therefore we can write

$T_{s}(E, t) \propto R_{s}(t) E^{-\mu_{s}(E)}$.

For SNRs $10^{15} \mathrm{~cm} \leq R_{s} \leq 10^{20} \mathrm{~cm} ; 10 \mu \mathrm{G} \leq H_{s} \leq 1 \mathrm{G}$; $10^{11} \mathrm{~cm} \leq l_{s} \leq 10^{16} \mathrm{~cm}$.

For such SNRs

$$
\begin{aligned}
& \mu_{s}(E)=0 \text { for } E \leq 3 \times 10^{13} \mathrm{eV}, \\
& \mu_{s}(E)=\mu_{s} \text { for } 3 \times 10^{13} \mathrm{eV}<E \leq 3 \times 10^{17} \mathrm{eV}, \\
& \mu_{s}(E)=0 \text { for } E>3 \times 10^{17} \mathrm{eV} .
\end{aligned}
$$

Usually, four stages of SNR evolution are considered. During the first three stages the SNR radius increases with time as (Gorbatskii 1977; Lozinskaia 1986; Pacini \& Salvati 1973; Shklovskii 1976; Woltjer 1972)

$R_{s} \propto t^{\varepsilon(t)}$, where $\varepsilon=1, \varepsilon=2 / 5, \varepsilon=1 / 4$ for stages I, II, III. The fourth stage of SNR evolution is not considered as the SNR dissipates in this stage into the interstellar medium.

From (29)-(31)

$T_{s}(E) \propto t^{\varepsilon(t)} E^{-\mu_{s}(E)}$.

Now we consider the evolution of the cosmic-ray spectrum through acceleration in the pulsar and later on in the supernova remnant. The particle spectrum from acceleration in the pulsar magnetosphere is (Crutcher et al. 1999)

$n_{s}(E, t) \propto t^{-\alpha} E^{-\gamma_{\mathrm{o}}}$

where $t$ is the lifetime of the pulsar and $\alpha$ is the pulsar luminosity index (for pulsar NP 0532 in the Crab nebula $\alpha \approx 2.3$ ).

This particle spectrum transforms due to propagation both in the SNRs and the interstellar medium. According to Eq. (25), for our model this spectrum looks like

$n_{\mathrm{G}}(E, t) \propto N_{s}\left(R_{i}(t)\right) n_{s}(E, t) \frac{V_{s}\left(R_{i}(t)\right)}{V_{\mathrm{G}}} \frac{T_{\mathrm{G}}(E)}{T_{s}(E, t)}$.

The numbers of SNRs in the Galaxy are (Lozinskaia 1986; Shklovskii 1976; Ilovaisky \& Legueux 1972; Pacini \& Salvati 1973; Woltjer 1972)

$N_{s}\left(R_{s}(t)\right) \propto R_{s}^{1 / \varepsilon(t)} \backsim t$.

The SNR volume

$V_{s}\left(R_{s}(t)\right)=4 \pi R_{s}^{3}(t) / 3 \backsim t^{3 \varepsilon(t)}$.

From Eqs. (32)-(36) we obtain

$n_{\mathrm{G}}(E, t) \propto t^{1-\alpha+2 \varepsilon(t)} E^{-\gamma_{\mathrm{o}}-\mu_{\mathrm{G}}(E)+\mu_{s}(E)}$.

For the above parameters $\alpha, \varepsilon, \mu_{\mathrm{G}}, \mu_{\mathrm{SNR}}$ the cosmic ray concentrations are

$n_{\mathrm{IG}}(E, t) \propto t^{0,7} E^{-\gamma(E)}$

$n_{\mathrm{IIG}}(E, t) \propto t^{-0,5} E^{-\gamma(E)}$

$n_{\mathrm{IIIG}}(E, t) \propto t^{-0,8} E^{-\gamma(E)}$,

respectively for stages I, II, III.

Here

$\gamma(E)=\gamma_{\mathrm{o}} \quad$ for $E \leq 3 \times 10^{13} \mathrm{eV}$,

$\gamma(E)=\gamma_{\mathrm{o}}-\mu_{s} \quad$ for $3 \times 10^{13} \mathrm{eV}<E \leq 3 \times 10^{15} \mathrm{eV}$,

$\gamma(E)=\gamma_{\mathrm{o}}-\mu_{s}+\mu_{\mathrm{G}}$ for $3 \times 10^{15} \mathrm{eV}<E \leq 3 \times 10^{17} \mathrm{eV}$,

$\gamma(E)=\gamma_{\mathrm{o}} \quad$ for $E>3 \times 10^{17} \mathrm{eV}$.

It follows from Eqs. (38) and (39) that the cosmic-ray spectrum in the interstellar medium will change essentially due to propagation in the Galaxy. If the initial cosmic ray spectrum in the sources has a constant power-law index, then in the interstellar medium this spectrum transforms due to propagation with the power-law index depending on energy. The cosmic ray spectrum at energies $E \leq 3 \times 10^{13} \mathrm{eV}$ and $E>3 \times 10^{17} \mathrm{eV}$ does not change due to propagation in SNR and in the interstellar medium. Cosmic rays with energy $3 \times 10^{13} \mathrm{eV}<E \leq 3 \times 10^{15} \mathrm{eV}$ change their spectrum due to their propagation in supernova remnants. For cosmic rays with 
energy $3 \times 10^{15} \mathrm{eV}<E \leq 3 \times 10^{17} \mathrm{eV}$ the spectrum changes due to their propagation both in SNRs and in the interstellar medium.

The parameters $\gamma_{\mathrm{o}}, \mu_{\mathrm{G}}, \mu_{s}$ may be estimated using experimental data about the cosmic ray spectrum. The experimental cosmic ray spectrum in near-Earth space is (Abu-Zayyad et al. 2001; Apanasenko et al. 2001a; Apanasenko et al. 2001b; Berezinskii et al. 1984; Dorman 1975; Dova et al. 2001; Fowler et al. 2001; Gaisser et al. 2001; Ginzburg \& Syrovatskii 1963; Hillas 1974; Kempa et al. 1974; Muraishi et al. 2001; Tkaczyk 2001)

$n(E) \propto E^{-\gamma(E)}$,

where

$$
\begin{array}{ll}
\gamma(E)=2.7 & \text { for } E \leq 3 \times 10^{13} \mathrm{eV}, \\
\gamma(E)=2.4-2.7 & \text { for } 3 \times 10^{13} \mathrm{eV}<E \leq 3 \times 10^{15} \mathrm{eV}, \\
\gamma(E)=3.4 & \text { for } 3 \times 10^{15} \mathrm{eV}<E \leq 3 \times 10^{17} \mathrm{eV}, \\
\gamma(E)=2.7 & \text { for } E>3 \times 10^{17} \mathrm{eV} .
\end{array}
$$

From Eqs. (38)-(41) we obtain $\gamma_{o}=2.7, \mu_{\mathrm{G}}=1, \mu_{s}=0.0-0.3$.

Comparison of the theoretical spectrum (38) and the experimental spectrum (40) shows that these spectra agree well. Both spectra have the same values of energy $E_{1} \approx 3 \times 10^{13} \mathrm{eV}$, $E_{2} \approx 3 \times 10^{15} \mathrm{eV}, E_{1} \approx 3 \times 10^{17} \mathrm{eV}$ where the power index of the spectrum changes. This fact supports our model of the propagation and formation of the cosmic ray spectrum in the Galaxy.

\section{Conclusions}

From our results we can draw the following conclusions.

1. The initial cosmic-ray spectrum in sources changes due to propagation both in the sources and the interstellar medium. If the initial cosmic-ray spectrum in the sources is a power-law with constant index $\gamma_{o}$, then later on this spectrum transforms due to propagation in the interstellar medium. The power-law index now depends on the particle energy $\gamma=\gamma(E)$. The spectrum for cosmic rays with energy $E \leq 3 \times 10^{13} \mathrm{eV}$ and $E>3 \times 10^{17} \mathrm{eV}$ does not change due to propagation either in SNRs or the interstellar medium since in these ranges the cosmic-ray lifetime does not depend on energy either for SNR or the Galaxy. For cosmic rays with energy $3 \times 10^{13} \mathrm{eV}<E \leq$ $3 \times 10^{15} \mathrm{eV}$ the spectrum changes due propagation in SNRs. For cosmic rays with energy $3 \times 10^{15} \mathrm{eV}<E \leq 3 \times 10^{17} \mathrm{eV}$ the spectrum will change due to propagation both in SNRs and in the interstellar medium. As a result, the cosmic ray spectrum near Earth differs from the spectrum in the sources and a knee will arise in the energy range $E \propto 10^{14}-10^{17} \mathrm{eV}$. We observe just such a knee in the cosmic ray spectrum near Earth.

2. The cosmic-ray lifetime in the Galaxy decreases to $10^{5}$ years from $10^{8}$ years (in the standard model) due to scattering on H-II regions. This result requires an increase in the source power for Galactic cosmic rays from $3 \times 10^{40} \mathrm{erg} / \mathrm{s}$ (in the Ginzburg-Syrovatskii model) to $10^{42} \mathrm{erg} / \mathrm{s}$.

3. The cosmic rays lose energy by interaction with SNRs, $\mathrm{SW}$ and $\mathrm{H}-\mathrm{II}$ regions due to adiabatic cooling. These losses are $3 \times 10^{39} \mathrm{erg} / \mathrm{s}$ and are comparable with losses due to nuclear interactions of cosmic rays with interstellar gas $\left(3 \times 10^{38}-3 \times\right.$ $\left.10^{39} \mathrm{erg} / \mathrm{s}\right)$. It is necessary to take them into account in models of cosmic-ray propagation in the Galaxy.

Evidently, the SNRs and H-II regions influence the formation of the cosmic-ray spectrum and the energy balance of cosmic rays in the Galaxy.

\section{References}

Abu-Zayyad, T., Belov, K., Bird, D. J., et al. 2001, ApJ, 556, 686 Allen, G. E., Petre, R., \& Gotthelf, E. V. 2001, ApJ, 558, 739

Apanasenko, A. V., Beresovskaya, V. A., Fujii, M., et al. 2001a, Proton and Helium spectra observed by RUNJOB, in Proc. 27th Intern. Cosmic Ray Conf., Hamburg, Germany, 07-15 Aug. 2001, 1626

Apanasenko, A. V., Sukhadolskaya, V. A., Derbina, V. A., et al. 2001b, Astropart. Phys., 16, 13

Berezinskii, V. S., Bulanov, S. V., Ginzburg, V. L., et al. 1984, The astrophysics of cosmic rays (Moscow: Izdatel'stvo Nauka, in Russian)

Bourke, T. L., Myers, P. C., Robinson, G., et al. 2001, ApJ, 554, 916 Brogan, C. L., Frail, D. A., Goss, W. M., et al. 2000, ApJ, 537, 875

Chandrang, B. D. G. 2000, ApJ, 529, 513

Clay, R. W. 2000, Publ. Astron. Soc. Aust., 17, 212

Clay, R. W. 2001, Publ. Astron. Soc. Aust., 18, 148

Clay, R. W. 2002, Publ. Astron. Soc. Aust., 19, 228

Crutcher, R. M., Roberts, D. A., Troland, T. H., et al. 1999, ApJ, 515, 275

Dickel, J. R., Milne, D. K., \& Strom, R. G. 2000, ApJ, 543, 840

Dorman, L. I. 1975, Experimantal and theoretical fundamentals of the astrophysics of cosmic-ray (Moscow: Izdatel'stvo Nauka, in Russian)

Dova, M. T., Epele, L., \& Swain, J. D. 2001, Explanation of the knee in the CR spectrum via interaction with the massive neutrinos in the Halo, in Proc. 27th Intern. Cosmic Ray Conf., Hamburg, Germany, 07-15 Aug. 2001, 1780

Erlykin, A. D., \& Wolfendale, A. W. 2001, AdSpR, 27, 803

Erlykin, A. D., \& Wolfendale, A. W. 2000, A\&A, 356, L63

Fowler, J. W., Fortson, L. F., Jui, C. C. H., et al. 2001, Astropart. Phys., 15,49

Gaisser, T. K., Honda, M., Lipari, P., et al. 2001, Primary spectrum to $1 \mathrm{TeV}$ and beyond, in Proc. 27th Intern. Cosmic Ray Conf., Hamburg, Germany, 07-15 Aug. 2001, 1643

Ginzburg, V. L., \& Syrovatskii, S. I. 1963, Origin of cosmic rays (Moscow: Izdatel'stvo AN USSNR, in Russian), (English: Ginzburg, V. L., Syrovatskii, S. I. 1964, The Origin of Cosmic Rays, New York: Macmillan)

Gorbatskii, V. G. 1977, Space gasdynamics (Moscow: Izdatel'stvo Nauka, in Russian)

Gray, A. D., Landecker, T. L., Dewdney, P. E., et al. 1999, ApJ, 514, 221

Habing, H. J. 1972, Cosmic electrodynamics (Moscow: Izdatel'stvo Mir, in Russian)

Hillas, A. M. 1974, Phil. Trans. Roy. A, 277, 413

Honda, M. 1987, ApJ, 319, 836

Hundhausen, A. J. 1976, Coronal expansion and solar wind (Moscow: Izdatel'stvo Mir, in Russian)

Houston, B., Riley, P. A., \& Wolfendale, A. W. 1983, Space Sci. Rev., 36

Ilovaisky, S. A., \& Legueux, J. A. 1972, A\&A, 20, 347

Jonis, F. C., Lukasiak, A., \& Ptuskin, V. 2001, ApJ, 547, 264

Jun Byung, I., \& Norman, M. L. 1996, ApJ, 472, 245

Kempa, J., Wdowzyk, J., \& Wolfendale, A. W. 1974, J. Phys. A, 7, 1213 
Kryvdyk, V. G. 1978, Phys. Solariterr. (Potsdam), 8, 21

Kryvdyk, V. G. 1978, Astrofizika (Armenia), 14, 257

Lacey, C. K., \& Duric, N. 2001, ApJ, 560, 719

Li, T. P., Riley, P. A., \& Wolfendale, A. W. 1983, MNRAS, 203, 87

Lozinskaia, T. A. 1986, Supernovae and stellar wind: interaction

Muraishi, H., Yanagita, S., \& Yoshida, T. 2001, Origin of the knee in the cosmic ray spectrum, in Proc. 27th Intern. Cosmic Ray Conf., Hamburg, Germany, 07-15 Aug. 2001, 1995

Tkaczyk, W. 2001, Is the knee and ankle in cosmic ray spectrum due to its propagation, in Proc. 27th Intern. Cosmic Ray Conf., Hamburg, Germany, 07-15 Aug. 2001, 1979
Pacini, F., \& Salvati, M. 1973, ApJ, 186, 249

Schleuning, D. A., Vaillancourt, J. E., Hildebrand, R. H., et al. 2000, ApJ, 535, 913

Shklovskii, I. S. 1976, Supernova stars and problems associated with them, 2nd revised and enlarged edition (Moscow: Izdatel'stvo Nauka, in Russian)

Sobolev, V. V. 1985, Course in theoretical astrophysics, 3rd revised and enlarged edition (Moscow: Izdatel'stvo Nauka, in Russian)

Strong, A. W., \& Moskalenko, I. V. 1998, ApJ, 509, 212

Tsao, C. H., Silberberg, R., \& Barghouty, A. F. 2001, ApJ, 549, 320

Woltjer, L. 1972, ARA\&A, 10, 129 